\title{
Surgical treatment of abdominal paragangliomas
}

\author{
Sadegh Toutounchi', Małgorzata E. Legocka', Ryszard Pogorzelski', Łukasz Zapała' ${ }^{2}$, Ewa Krajewska', \\ Krzysztof Celejewski', Urszula Ambroziak', Zbigniew Gałązka ${ }^{1}$
}

${ }^{1}$ Department of General, Endocrinological and Vascular Surgery, Medical University of Warsaw, Warsaw, Poland

${ }^{2}$ Department of General, Oncological and Functional Urology, Medical University of Warsaw, Warsaw, Poland

${ }^{3}$ Department of Internal Medicine and Endocrinology, Medical University of Warsaw, Warsaw, Poland

\begin{abstract}
Introduction: Extraperitoneal, abdominal paragangliomas most commonly originate from the sympathetic nervous system. Typical features include catecholamine overproduction and the potential for malignancy. Lesions are usually located paravertebrally, but when growing in a more expansive manner they may also appear between the inferior vena cava and aorta. In the authors' opinion this site excludes laparoscopic tumourectomy.

Material and methods: Twenty-eight patients were selected for surgical management of abdominal paragangliomas in the past eight years at our endocrine surgical centre. This group consisted of 21 (75\%) women and seven (25\%) men, aged 14 to 84 years (mean 47.9$)$. In $13(46.4 \%)$ cases paroxysmal hypertension was observed. Type 2 diabetes was noted in another $10(35.7 \%)$ patients, and Takotsubo acute coronary syndrome in two $(7.1 \%)$. Patients were preoperatively qualified for either open surgery or laparoscopic tumourectomy based on visualisation and location of the tumours in imaging studies.

Results: All patients were successfully operated. Eleven (39.3\%) patients qualified for laparoscopy, while the remaining 17 (60.7\%) were treated with an open surgical approach due to difficult access to the lesion. The mean operative time was 130 minutes for laparoscopy and 120 minutes for laparotomy $(\mathrm{p}=0.2)$. There were no local or general complications after either type of procedure.

Conclusions: The use of laparoscopic access is practically excluded in the treatment of paragangliomas located between the inferior vena cava and aorta, especially at the level of the renal vessels and extending superiorly to the diaphragm. (Endokrynol Pol 2019; 70 (6): 469-472)

Key words: paragangliomas; pheochromocytoma; surgery of paragangliomas
\end{abstract}

\section{Introduction}

Paragangliomas may arise in practically all locations in the human body in which ganglion or argyrophilic tissue are present. In the majority of cases, they surround tissues of the central nervous system. The frequency of appearance in abdominal and pelvic areas is estimated to be approximately $21 \%$. The exact location of paraganglioma influences its natural history, symptomatology, hormonal overactivity, and potential for malignancy. Abdominal paragangliomas arise from sympathetic ganglia and often have a tendency towards hormonal overproduction and malignancy, in comparison to other locations. The main symptoms are an abnormal mass in the abdominal cavity, hypertension, and abdominal pain [1]. In $25 \%$ of cases they are a part of multiple endocrine syndromes and express germinal mutations in the following genes: RET, VHL, SDHB, and SDHD [2].

\section{Material and methods}

Over the past eight years there were 440 patients operated due to adrenal disease in our clinic, of whom $28(6.4 \%)$ were due to abdominally located paragangliomas. This group consisted of 21 (75\%) women and seven (25\%) men, aged 14-82 years (mean 47.9). These patients were analysed according to clinical symptoms, tumour location, method of surgical treatment, histopathological tissue analysis, and other criteria.

\section{Symptomatology and diagnostics}

Thirteen of $28(46.4 \%)$ patients underwent initial diagnostics due to hypertension, while the remaining $15(53.6 \%)$ remained asymptomatic. In these latter cases, the presence of paraganglioma was confirmed incidentally (incidentaloma) during imaging studies performed for other indications. Hormonal overproduction defined by the presence of catecholamines in plasma was discovered in seven (25\%) patients. Diagnosis was confirmed based on increased levels of metanephrine in 24-hour urine collection in all these cases, as well as two additional cases out of nine (32.1\%). In $10(35.7 \%)$ patients Takotsubo-like acute coronary syndrome had previously been reported. All endocrinological laboratory diagnostics were carried out in the internal medicine and endocrinology departments. Based on imaging studies, it seems that ultrasound (US) is of key importance as a screening test. While analysing the diagnostic course of particular patients from the study group, it was discovered that US was performed two to five times in each patient in order to assess tumour growth dynamics. Computed tomography (CT) was performed in all cases to estimate the topography of the tumour and its relation to adjacent structures (Fig. 1, 2). In several cases, confirmatory imaging was by magnetic resonance. I-MIGB and FDG-PET were also conducted in a few clinical scenarios, 


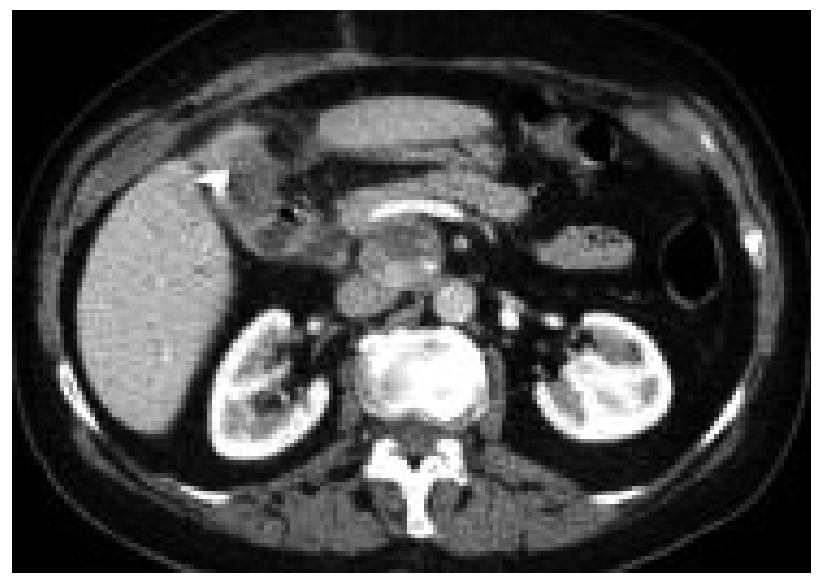

Figure 1. Patient P.J., aged 76 years. Paraganglioma located between inferior vena cava and aorta, below pancreas and splenic artery, in computed tomography

Table I. The tumour spread based on authors' material

\begin{tabular}{lcc}
\hline Location & No. & $\%$ \\
\hline Organ of Zuckerkandl & 6 & 21.4 \\
\hline Between IVC and aorta & 7 & 25.1 \\
\hline Below renal vessels & 5 & 17.8 \\
\hline Below IVC & 4 & 14.3 \\
\hline In the proximity of adrenals & 6 & 21.4 \\
\hline Overall & 28 & 100.0 \\
\hline
\end{tabular}

especially in those patients with multiple lesions, or when tumour location was ambiguous.

\section{Location}

The present study focused only on abdominal paragangliomas (subdiaphragmatic location). The tumour spread based on our material is presented in Table I.

In cases involving tumours of the organ of Zuckerkandl, three patients had tumours on the anterior aspect of the aorta found in imaging studies (three tumours in all cases).

Tumours located between the inferior vena cava and aorta, especially at levels beginning from the aortic bifurcation and extending to the diaphragmatic crura, remain challenging from a surgical point of view. The majority of these cases excluded laparoscopic tumourectomy. The most difficult sites in particular involve tumours located retropancreatically, as well as those superior and adjacent to the diaphragmatic crura. Lesions located below the right renal vessels and inferior vena cava allow for laparoscopic tumourectomy, which is usually limited due to the proximity to the left adrenal gland. In order to remove a paraganglioma, it is often necessary to excise it en bloc with the right adrenal gland, due to surgical inability to precisely identify the border between the adrenal gland and paraganglioma.

It is impossible to separate paravertebrally located paragangliomas in most cases where the paraganglioma is in close relation with the left adrenal gland (Fig. 3). There are some situations, in which a different surgical approach or even conversion to open surgical technique is unavoidable.

\section{Treatment}

Due to high risk of hormonal hyperactivity of the tumour, 21 (75\%) patients were treated with alpha-blockers at a total dose of 6-12 mg

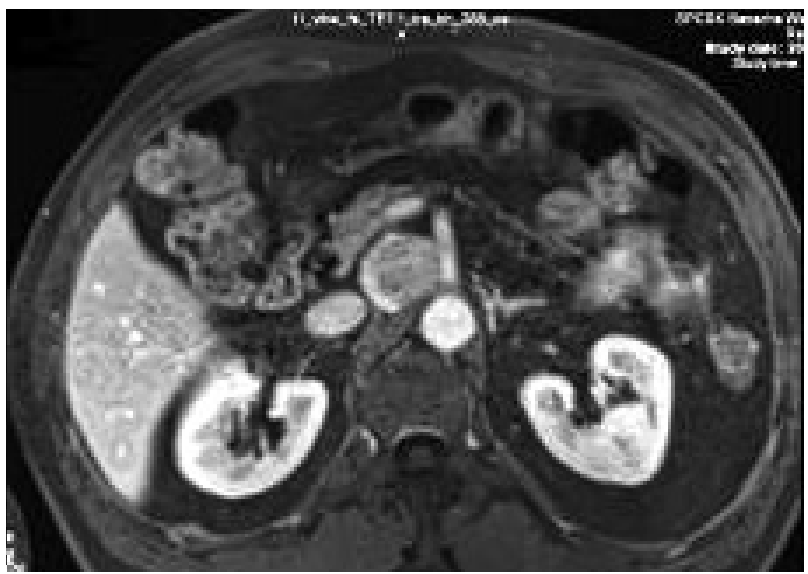

Figure 2. Patient M.E., aged 61 years. Paraganglioma adjacent to celiac artery, in magnetic resonance imaging

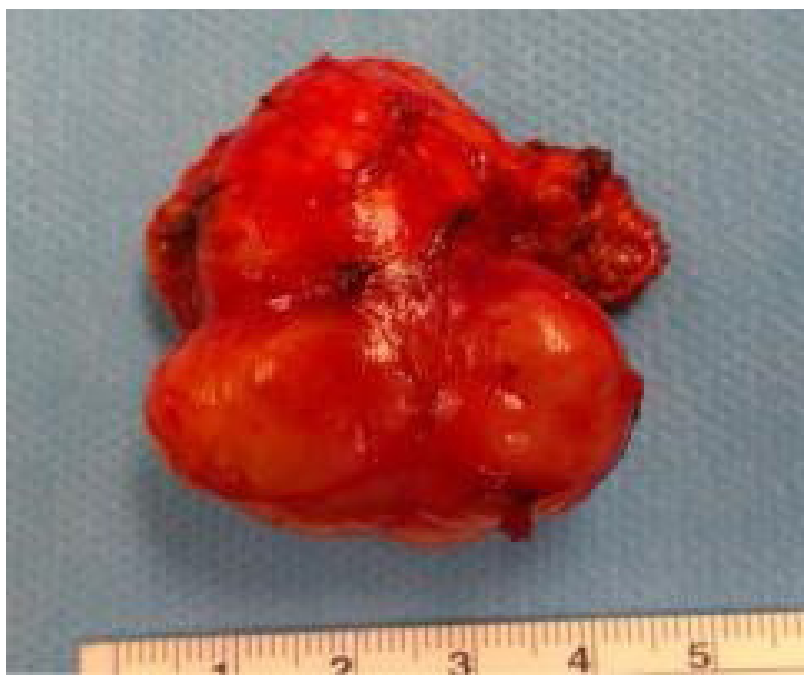

Figure 3. Patient L.E. aged 73 years. Paraganglioma specimen excised together with adrenal gland. Paraganglioma visible in the foreground, with adjacent normal adrenal gland superiorly

per day. This treatment was introduced around two to four weeks before scheduled surgery. In the remaining seven $(25 \%)$ patients, alpha blockers were not used.

After pharmacological preoperative treatment and exact localisation and analysis of the lesions, all patients were operated. Precise analysis of location determined qualification for laparoscopic procedure in $11(39.3 \%)$ patients. The remaining $17(60.7 \%)$ were prepared for laparotomy. Indications for laparoscopy included: close proximity to adrenal glands, renal vessels bilaterally or paravertebrally below inferior vena cava on the right.

\section{Results}

Lateral transperitoneal laparoscopic approach was performed in 11 patients, using four ports. Two of these patients required conversion to open surgery due to difficult tumour location. Paragangliomas were located proximal to both left and right adrenal glands in six patients, usually between the adrenal gland and 
vertebral column, or below the lower pole of the adrenal gland. Adrenalectomy was performed when separation of the adrenal gland from the paraganglioma was impossible. The mean operating time was 130 minutes (range 75-210 minutes).

The remaining 17 patients qualified for open surgery. Depending on tumour location, several different access points were used: midline incision in six cases, bilateral paramedian incision in six cases, and Kocher's incision and lumbotomy in the remaining five cases. In those cases, the mean operating time was 120 minutes (range 75-150 minutes).

Blood pressure spiked to values over 200/100 mm Hg in 13 cases, regardless of surgical method. This necessitated pausing the procedure and administering additional hypotensive agents until blood pressure stabilised.

Intraoperative fluctuations of blood pressure were present in 11 patients who underwent preoperative treatment with $\alpha$-blockers and in two patients without this pre-treatment.

A sudden drop in blood pressure was observed in three patients after removal of the paraganglioma, which was managed with high doses of amines. All of these patients had preoperatively received $\alpha$-blockers at a dose of $12 \mathrm{mg}$ per day.

The mean stay in hospital was six days, irrespective of surgical method. There were no cases of local or organ complications postoperatively. During the early postoperative period, normalisation of blood pressure was observed in $10(76.9 \%)$ of the 12 patients with hypertension. In those cases, continuation of hypotensive therapy was not needed. A similar situation was noted in the group of 10 patients with type 2 diabetes. Normoglycaemia was achieved in eight patients $(80 \%)$ postoperatively, allowing for cessation of hypoglycaemic agents.

The types of lesions found in histopathological studies of surgical specimens are presented in Table II.

Table II. The types of lesions found in histopathological studies of surgical specimens

\begin{tabular}{lcc}
\hline Pathological results & $\begin{array}{c}\text { Number } \\
\text { of patients }\end{array}$ & $\begin{array}{c}\text { Percentage } \\
(\%)\end{array}$ \\
\hline Paraganglioma* & 22 & 78.6 \\
\hline Ganglioneuroma & 4 & 14.3 \\
\hline Schwannoma & 2 & 7.1 \\
\hline Overall & 28 & 100.0 \\
\hline
\end{tabular}

* In two patients, there were three paragangliomas resected from the anterior wall of the aorta and left lateral surface of the vertebral column.

Another two patients had a history of recurring paragangliomas after previous abdominal surgery. Present long-term follow-up includes 23 (82.1\%) patients.

The remaining five patients were lost to follow-up.

\section{Discussion}

The frequency of paragangliomas in the general population is estimated to be $1-8$ new cases per one million. They are most commonly located in the central nervous system. Another $21 \%$ of paragangliomas are located within the abdominal cavity. In this location they mainly present in ganglions of the sympathetic nervous system $[1,3]$, most often in the sympathetic trunk at the lateral surface of the vertebral column. Other, more infrequent sites involve the neck, chest, and urinary bladder.

When growing expansively, they may appear between the inferior vena cava and aorta, bilaterally below the renal vessels, and at the anterior aspect of the aortic wall between the inferior mesenteric artery and aortic bifurcation (organ of Zuckerkandl) [4]. Disease presentation in our study reflected this tendency. Paragangliomas located in proximity to the adrenal glands pose a clinical problem, especially when they are located between the vertebral column and adrenals. In six cases the authors were forced to perform adrenalectomy together with ganglion resection. Right-sided removal of the paraganglioma usually involved ligation of the adrenal vein, which in turn led to unavoidable adrenalectomy. On the left side, direct contact between the paraganglioma and adrenal gland made separation of these structures impossible.

In over $50 \%$ of cases, diagnosis of paraganglioma is related to a thorough work-up of paroxysmal hypertension. The remaining cases are usually found by chance due to imaging of other abdominal organs (incidentaloma). More infrequently, clinical indications such as abdominal pain or palpable abdominal mass [4, 5] lead to specific diagnostic imaging and discovery of paraganglioma. In the presented material paroxysmal hypertension was found in $46.4 \%$ of patients. Laboratory tests performed during patients' hospitalisation in internal medicine or endocrine departments led to the diagnosis in all cases. Additionally, four of our patients (14.3\%) had mutations in the RET gene. Other authors reported genetic mutations in paragangliomas in seven $(26.7 \%)$ cases.

Surgical treatment of paragangliomas is indicated in all cases because even long-term asymptomatic surveillance may be misleading. Additionally, catecholamine overproduction may cause sudden and dangerous elevations in blood pressure. Catecholamine release into the bloodstream may be triggered by any stressful situation. Currently laparoscopy, by both trans- and retroperitoneal access [6-8], is the surgical treatment of choice. This is feasible only in cases where paragangliomas are located: along with sympathetic ganglions inferiorly to the renal vessels, in proximity to the renal hilum, and in some cases originating from the organ of 
Zuckerkandl. In the authors' experience, paragangliomas adjacent to the inferior vena cava and aorta, especially at the level of renal vessels and below, are not possible to be removed via laparoscopic approach. Tumours located at the level of the pancreas or diaphragmatic crura may result in serious difficulties even in open surgery. Because they are well-vascularised structures (aortic inflow and inferior vena caval outflow), their excision, especially at these locations, may result in dramatic intraoperational haemorrhage. Their vessels are usually short, and bleeding may be stopped only after underpinning the wall of the aorta or inferior vena cava, which is extremely difficult during laparoscopic approach. The authors qualified 11 (39.3\%) patients for surgery by primary laparoscopic technique, while the remaining $17(60.7 \%)$ were operated via open access due to inaccessible tumour location.

In our material, the post-surgical histopathological reports confirmed paragangliomas in $22(78.6 \%)$ patients. The remaining six cases $(21.4 \%)$ proved to be other lesions (four ganglioneuroma; two schwannoma). Similar results were obtained by different authors [7]. In six patients the location of the lesion was within the organ of Zuckerkandl. This site is connected with high metastatic risk in up to 66\% [9] of cases. Taking into consideration these data and the malignant potential of paragangliomas, which is estimated to be between 10 and $35 \%$, all patients with paragangliomas require strict follow-up during the postoperative period [10].

\section{Conclusions}

Laparoscopy can be feasible and safe in the treatment of paragangliomas, depending on the location of the tumour. The authors believe that tumours located between the inferior vena cava and aorta, especially at the level of the renal vessels and extending superiorly to the diaphragm, should be considered as a contraindication to laparoscopic access.

\section{References}

1. Fischer T, Gaitonde S, Jones M, et al. Anatomic Location Is the Primary Determinant of Survival for Paragangliomas. Am Surg. 2017; 83(10): 1132-1136, indexed in Pubmed: 29391110.

2. Krawczyk A, Hasse-Lazar K, Pawlaczek A, et al. Germinal mutations of RET, SDHB, SDHD, and VHL genes in patients with apparently sporadic pheochromocytomas and paragangliomas. Endokrynol Pol. 2010; 61(1): 43-48, indexed in Pubmed: 20205103.

3. Pacak K. Pheochromocytoma and paraganglioma. www.endotext. org (07.04.2018).

4. Ji XK, Zheng XW, Wu XL, et al. Diagnosis and surgical treatment of retroperitoneal paraganglioma: A single-institution experience of 34 cases. Oncol Lett. 2017; 14(2): 2268-2280, doi: 10.3892/ol.2017.6468, indexed in Pubmed: 28789448.

5. Gill T, Adler K, Schrader A, et al. Extra-adrenal pheochromocytoma at the organ of Zuckerkandl: a case report and literature review. Radiol Case Rep. 2017; 12(2): 343-347, doi: 10.1016/j.radcr.2016.12.009, indexed in Pubmed: 28491185.

6. Alemanno G, Bergamini C, Somigli R, et al. Abdominal paragangliomas: a quantitative prognostic score as predictive factor of the feasibility of the laparoscopic approach. Updates Surg. 2017; 69(4): 509-515, doi: 10.1007/s13304-017-0459-3, indexed in Pubmed: 28537037.

7. Abe T, Sazawa A, Harabayashi T, et al. Laparoscopic resection of paraaortic/paracaval neurogenic tumors: surgical outcomes and technical tips. Surg Endosc. 2016; 30(10): 4640-4645, doi: 10.1007/s00464-015-4740-6, indexed in Pubmed: 26715023.

8. Sho S, Yeh MW, Li N, et al. Single-incision retroperitoneoscopic adrenalectomy: a North American experience. Surg Endosc. 2017; 31(7): 3014-3019, doi: 10.1007/s00464-016-5325-8, indexed in Pubmed: 27826779 .

9. Ayala-Ramirez M, Feng L, Johnson MM, et al. Clinical risk factors for malignancy and overall survival in patients with pheochromocytomas and sympathetic paragangliomas: primary tumor size and primary tumor location as prognostic indicators. J Clin Endocrinol Metab. 2011; 96(3): 717-725, doi: 10.1210/jc.2010-1946, indexed in Pubmed: 21190975.

10. Kotecka-Blicharz A, Hasse-Lazar K, Handkiewicz-Junak D, et al. 131-MIBG therapy of malignant pheochromocytoma and paraganglioma tumors - a single-centre study. Endokrynologia Polska. 2018; 69(3): 246-251, doi: 10.5603/ep.a2018.0024, indexed in Pubmed: 29645065 . 